\title{
Voltage-Activated Potassium Channels in the Plasma Membrane of Rod Outer Segments: A Possible Effect of Enzymatic Cell Dissociation
}

\author{
S. Hestrin ${ }^{1}$ and J. I. Korenbrot ${ }^{1,2}$ \\ 'Department of Physiology and 'Department of Biochemistry, University of California, School of Medicine, San Francisco, \\ California 94143
}

\begin{abstract}
Using patch-clamp techniques, we recorded single-channel currents from the plasma membrane of the outer segment of isolated light-adapted rods. The channels are potassiumselective and their conductance is about $87 \mathrm{pS}$. The channels are activated by depolarization and are not sensitive to cytoplasmic calcium, they are exclusively found in rods isolated with the proteolytic enzyme papain, and are not detected in rods isolated by mechanical means. Thus, these channels do not exist in an activatable form in the outer segment plasma membrane under physiological conditions. The channels might be derived from a normally inaccessible structure, such as the disk membrane, or, alternatively, they might be a modified form of a channel that is not active in the intact rod.
\end{abstract}

The photovoltage of rod photoreceptors exhibits a complex waveform that arises from the interaction of both light-sensitive and voltage-sensitive ionic channels (reviewed by Fain and Lisman, 1981; Korenbrot, 1985; and Schwartz, 1985). Studies of isolated rod inner segments have shown that some of the voltagc-sensitive but light-insensitive channels are localized in the plasma membrane of this region of the cell (Bader et al., 1982; Bader and Bertrand, 1984; Corey et al., 1984). In contrast, Baylor and Lamb (1982) have suggested that the plasma membrane of the outer segment contains only light-sensitive channels, since the total light-insensitive conductance of this membrane is no more than $10 \mathrm{pS}$. These results have been confirmed and extended to demonstrate that the light-insensitive conductance of the outer segment is not voltage-dependent (Baylor et al., 1984; Baylor and Nunn, 1986). The conclusion of these reports has recently been challenged by the studies of Kolesnikov et al. (1984), who reported that the outer segment of enzyme-dissociated rods contains voltage-activated channels that are lightinsensitive. The channels that Kolesnikov et al. (1984) discovered are chloride-selective and have a large unitary conductance of about 200 pS. Noell et al. (1982), Attwell and Gray (1984), and Sather et al. (1985) have also reported on the presence of light-insensitive, voltage-activated channels of appreciable size in the outer segment of rods.

To resolve this experimental discrepancy, we studied the lightinsensitive channels of the outer segment. We have used patch-

Received Aug. 12, 1986; revised Apr. 22, 1987; accepted Apr. 23, 1987.

We wish to thank Drs. Jeff Lansman and Erich Phillips for commenting on the manuscript. This work was supported by NIH Grants EY-01586, EY-05498, and a New Investigator Research Award, Grant EY NS-05617, to S.H.

Correspondence should be addressed to $S$. Hestrin at the above address.

Copyright (c) 1987 Society for Neuroscience $0270-6474 / 87 / 103072-09 \$ 02.00 / 0$ clamp techniques to record from single rods isolated from the tiger salamander retina. Our results suggest that the original proposition of Baylor and Lamb (1982) is correct and that the method of isolating and manipulating single rods can lead to the appearance of channels in the outer segment plasma membrane.

\section{Materials and Methods}

Materials. Tiger salamanders (Ambystoma tigrinum) at the larval stage were kept in an outdoor pool under continuous water flow or in 20 gallon tanks at $5^{\circ} \mathrm{C}$ under a $12 \mathrm{hr}$ dark-light cycle. They were fed earthworms once a week. Papain was purchased from Worthington (currently Cooper Bio-medical, Melvern, PA). Stock solution of minimum essential medium (MEM) amino acids and MEM vitamins were prepared at the UCSF tissue culture facility according to Gibco (Grand Island, NY; catalog nos. 320-1120 and 320-1130, respectively).

BAPTA (1,2-bis $\left(O\right.$-aminophenoxy)ethane- $N, N, N^{\prime}, N^{\prime}$-tetraacetic acid) was purchased from Molecular Probes (Eugenc, OR). The composition of all solutions used is listed in Tables 1 and 2 .

Enzymatic dissociation. The technique used of retinal dissociation with the proteolytic enzyme papain is similar to that reported by Bader et al. (1982). A retina was isolated from the eyecup of a dark-adapted animal under infrared illumination. In the light, the retina was transferred to a petri dish containing $4 \mathrm{ml}$ of solution $A_{\text {, }}$ to which $7 \mathrm{U} / \mathrm{ml}$ papain were added. The retina was incubated in the solution at room temperature for $30 \mathrm{~min}$. After the incubation, the retina was washed several times with solution $B$. The retina was then suspended in $1 \mathrm{ml}$ of solution B and mechanically triturated by repeated passage through the fire-polished end of a pasteur pipette. After trituration, $150 \mu \mathrm{l}$ of the cell suspension was applied to concanavalin A- (type IV; Sigma, St. Louis, MO) coated coverslips (Bader et al., 1982) held in a multiwell tray (Linbro, McLcan, VA). The cells were allowed to adhere to the substratum for $20 \mathrm{~min}$, after which solution $\mathrm{C}$ was added to the wells. The cells were maintained at $12^{\circ} \mathrm{C}$ and were used for experiments within $3 \mathrm{~d}$ after dissociation. The media were replaced daily.

Photoreceptors were also isolated by incubating the retinas overnight at $14^{\circ} \mathrm{C}$ in solution $\mathrm{C}$ with $7 \mathrm{U} / \mathrm{ml}$ papain. The results we obtained with both methods of cell isolation were indistinguishable.

Mechanical dissociation. The procedure that we used for isolating rods without enzymes is similar to the method described by Baylor et al. (1984). Cell dissociation was carried out in solution B. An isolated retina was positioned photoreceptor side up on a petri dish with a Sylgard- (Dow Corning, Midland, MI) coated bottom. A razor blade was used to chop the retina into fine pieces. The resulting suspension of retinal fragments and isolated cells was transferred onto concanavalin A-coated coverslips. The cells were allowed to adhere to the coverslip for $20 \mathrm{~min}$ and then solution $\mathrm{C}$ was added. These cells were studied within $1-5 \mathrm{hr}$ of the dissociation.

Electrical recording. A coverslip with the attached cells was positioned in a chamber mounted on the stage of an upright microscope equipped with differential interference contrast optics (Aus-Jena). The solution bathing the cells was then changed to a high-potassium solution (solution $\mathrm{D}$, Table 1). The concentration of calcium in this solution was sufficiently low to allow us to obtain stable inside-out patches of membrane when necessary. In some experiments, a fluoride-containing solution was used (solution E). 
Table 1. Extracellular solutions

\begin{tabular}{|c|c|c|c|c|c|c|c|c|c|c|c|}
\hline Sol. & $\mathrm{NaCl}$ & $\mathrm{KCl}$ & $\mathrm{K}$ asp. & $\mathrm{KF}$ & $\mathrm{CaCl}_{2}$ & $\mathrm{MgCl}_{2}$ & $\mathrm{NaHCO}_{3}$ & $\mathrm{CsCl}$ & $\begin{array}{l}\text { Glu- } \\
\text { cose }\end{array}$ & HEPES & MEM \\
\hline A & 95 & 2 & - & - & 2 & 2 & 5 & 1 & 10 & 10 & $100 \%$ \\
\hline B & 100 & 2 & - & - & 2 & 2 & 5 & - & - & 10 & $100 \%$ \\
\hline $\mathrm{C}$ & 95 & 2 & - & - & 2 & 2 & 5 & - & 10 & 10 & $100 \%$ \\
\hline $\mathrm{D}$ & - & 30 & 70 & - & 0.1 & 2 & 25 & - & - & - & $100 \% \%^{\alpha}$ \\
\hline E & - & 25 & - & 100 & - & 0.5 & - & - & - & 10 & - \\
\hline$F$ & 110 & 2 & - & - & 2 & 2 & - & - & - & 10 & - \\
\hline $\mathrm{G}$ & 2 & 110 & - & - & 2 & 2 & - & - & - & 10 & - \\
\hline
\end{tabular}

Concentrations are given in millimolars. Amino acids and vitamins prepared according to Gibco catalogs $320-1120$ and $320-1130$, respectively. $\mathrm{pH}$ was adjusted to 7.4 .

${ }^{a}$ Solution bubbled with $95 / 5 \mathrm{O}_{2} / \mathrm{CO}_{2}$ gas mixture.

Sylgard-coated patch-pipettes with tip openings of $1-0.5 \mu \mathrm{m}$ were used. For cell-attached recordings, the pipettes were filled with one of the solutions listed in Table 1, according to the purpose of the experiment. For whole-cell recordings (Hamill et al., 1981) the pipettes were filled with one of the solutions listed in Table 2. Currents were recorded with a patch-clamp amplifier (Yale Physiology Dept. Mark 4). The amplifier output was filtered with an 8-pole Bessel-type filter (model 902LPF; Frequency Devices, Haverhill, MA). The bandwidth of the filter was set at 0-5 kHz. A microcomputer (PDP 11/23; Digital Equipment, Maynard, MA) was used to acquire data and apply command voltages. Analog data were digitized at 12-bit accuracy (model DT2782; Data-Translation, Marlboro, MA) and stored on computer disks.

Data analysis. The mean amplitude of single-channel currents was obtained by averaging 20-50 single-channel events. Single-channel openings were detected by inspection of records displayed on the computer graphics terminal. The probability of a channel's being open was determined by integrating the current records made during an activating voltage pulse and dividing this quantity by the single-channel mean current. This method is reliable if the patch of membrane contains just one channel. We assumed that patches contained one channel only if there was no overlap of channel openings even when the overall channel activity was high.

\section{Results}

\section{Papain-isolated rods}

The enzyme dissociation procedure that we used yielded both intact rod photoreceptors and isolated rod inner segments. The experiments that we report here were performed on intact rods that appeared to have undamaged inner and outer segments. In most, but not all, cases, a prominent synaptic terminal was seen attached to the inner segment. Whole-cell recordings from these cells revealed voltage-dependent currents that were similar to those previously reported by Bader et al. (1982). Figure 1 illustrates a whole-cell recording from an intact rod with the pipette applicd to the inner scgment. The pipctte-filling solution in this experiment contained $5 \mathrm{~mm}$ BAPTA (solution $\mathrm{H}$ ) in order to maintain a low concentration of internal free calcium. The cell membrane was held at $-50 \mathrm{mV}$ and was depolarized to various levels. Depolarization evoked an inward and an outward current. The inward current was similar to the calcium current reported by Bader et al. (1982) and by Corey et al. (1984). The outward current was similar to the delayed rectifier reported by Bader et al. (1982). Membrane hyperpolarization from a holding potential of $-40 \mathrm{mV}$ activated an inward current similar to the inward rectifier reported by Bader and Bertrand (1984) and Hestrin (1987). The properties of the voltage-dependent currents were similar to those previously described by others in both enzyme-disociated cells (Bader et al., 1982; Bader and Bertrand, 1984; Corey et al., 1984) and mechanically isolated cells (Werblin, 1978; Attwell and Wilson, 1980; Hestrin, 1987). Thus, in our hands, the method of cell dissociation yielded rods that were functionally intact.

We obtained cell-attached patch-recording from the plasma membrane of the outer segment of enzymatically isolated rods. In this configuration, the absolute membrane potential is the sum of the applied voltage and the resting membrane potential Since the resting potential is likely to vary among cells, we minimized this difference by superfusing the cells with a potassium-rich solution (solution $\mathrm{G}$, Table 1). In the potassium-rich solution, the membrane potential is probably close to 0 and the voltage across the patch of membrane in the pipette is mainly determined by the voltage applied to the pipette. This assumption is supported by the fact that the behavior of single-channel currents measured from cell-attached patches in intact rods bathed in a high-K solution is very similar to that recorded in excised patches of membrane at the same applied membrane potential. Figure $2 A$ illustrates cell-attached recordings made with a pipette filled with a sodium-rich solution (solution F) from a rod bathed in a potassium-rich solution (solution $\mathrm{D}$ ). A holding voltage of $+50 \mathrm{mV}$ was applied to the pipette. Under these conditions, if we assume that the ccll's resting potential is $0 \mathrm{mV}$, the membrane potential of the patch would be -50 $\mathrm{mV}$. When the membrane potential of the patch was depolarized from the holding potential of $-50 \mathrm{mV}$ to potentials more positive than $0 \mathrm{mV}$, bursts of outward-going currents were observed. As shown in Figure $2 A$, the amplitude of these outward

\begin{tabular}{|c|c|c|c|c|c|c|c|c|c|}
\hline Sol. & $\mathrm{NaOH}$ & $\mathrm{KOH}$ & $\mathrm{KCl}$ & $\mathrm{MgCl}_{2}$ & ATP & GTP & BAPTA & EGTA & HEPES \\
\hline $\mathrm{H}$ & 25 & 40 & 15 & 2.5 & 2 & - & 5 & - & 80 \\
\hline I & - & 74 & 15 & 4 & 5 & 2 & - & 0.05 & 90 \\
\hline
\end{tabular}




\section{'whole-cell' recording}

Figure 1. Whole-cell current recorded from an enzyme-isolated intact rod. The patch-pipette was filled with solution $\mathrm{H}$ and was sealed on the inner segment. The cell was depolarized to $-30,-20$, $-15,-10,-5,0$, and $+5 \mathrm{mV}$, from a holding potential of $-50 \mathrm{mV}$. The membrane potential is given as the pipette voltage in reference to the bath voltage.
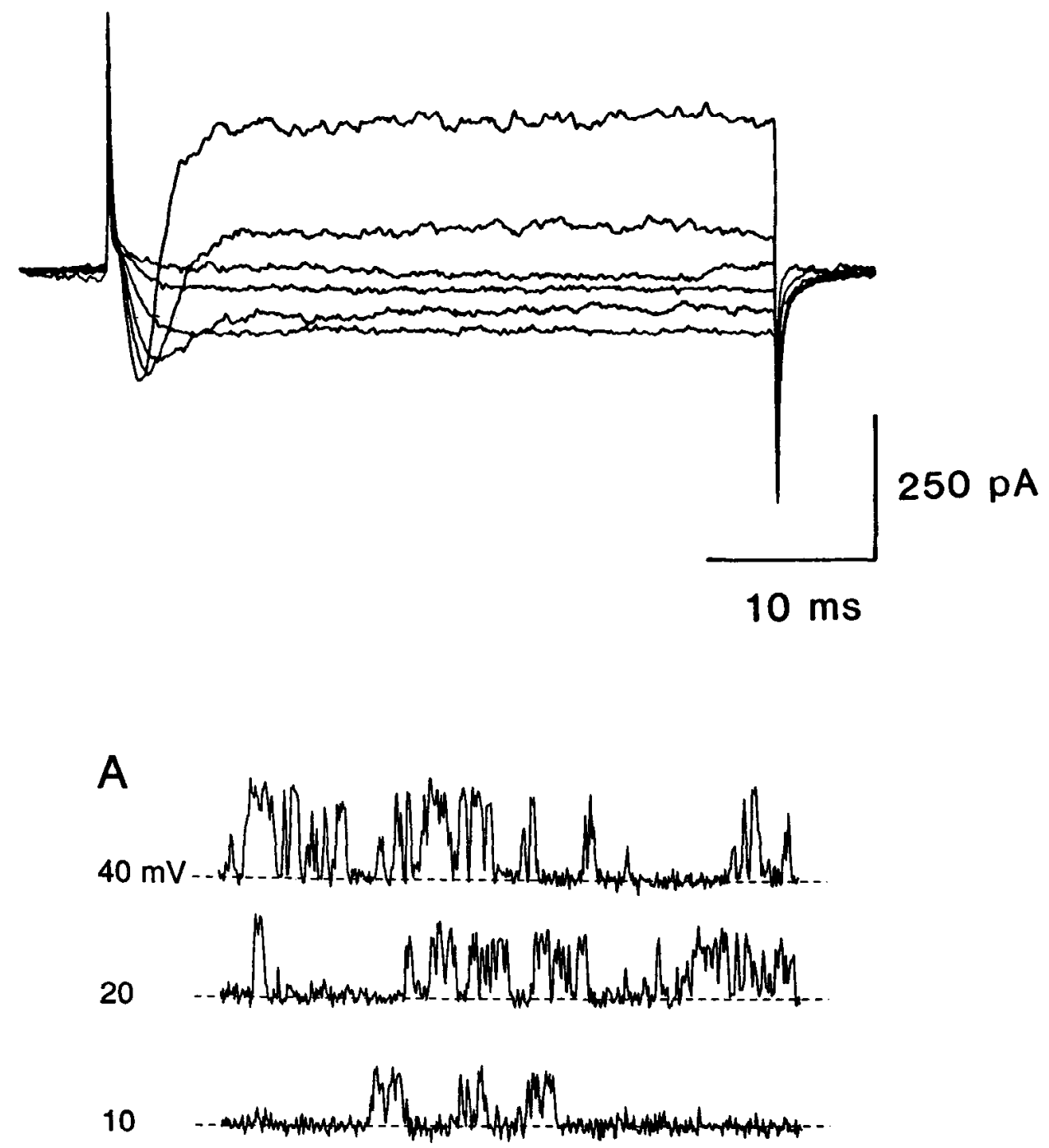

Figure 2. Voltage-activated, singlechannel currents measured from cellattached membrane patches of the outer segment of papain-isolated rods. $A$, The cells were bathed in potassium-rich solution (solution D). The pipette was filled with solution $F$. The negative of the voltage applied to the pipette, in reference to the bath voltage, is marked on the left of each trace. The cell's resting membrane potential was probably about $0 \mathrm{mV}$ because the bath solution was potassium-rich. Voltage steps that hyperpolarized the membrane patch in reference to the bath did not produce channel activity. $B$, The cells were bathed in a sodium-rich solution (solution C). The patch-pipette was filled with solution $F$. The negative of the voltage that was applied to the pipette is marked on the left of each trace. The actual membrane potential of this patch was more negative than the marked voltage by an amount equal to the cell's resting potential.

B

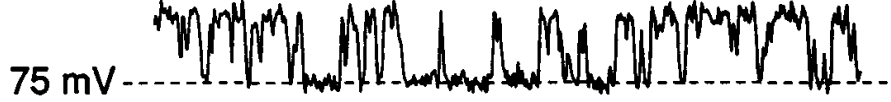
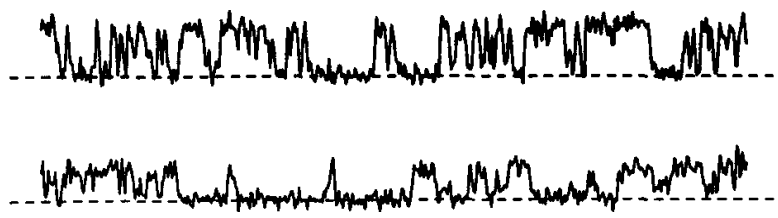


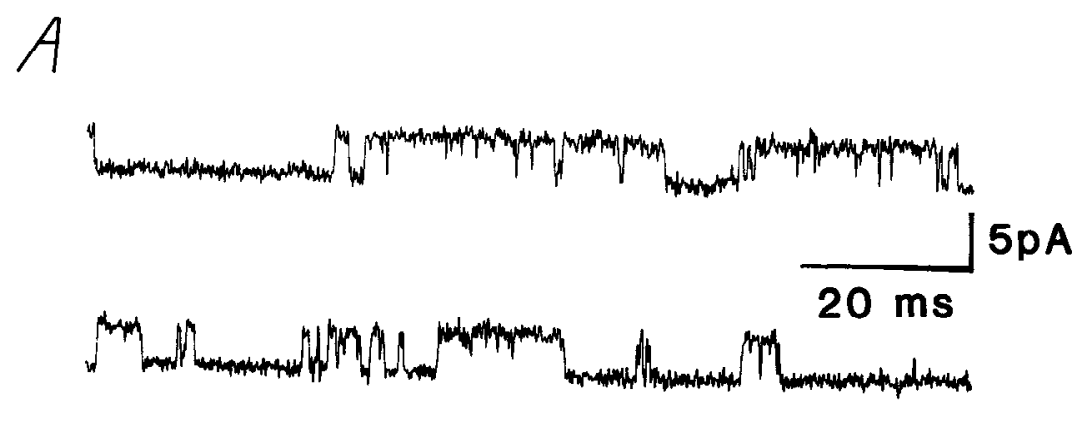

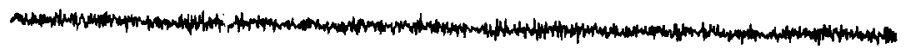

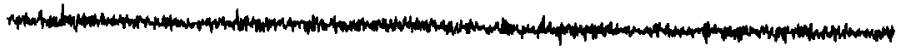

G

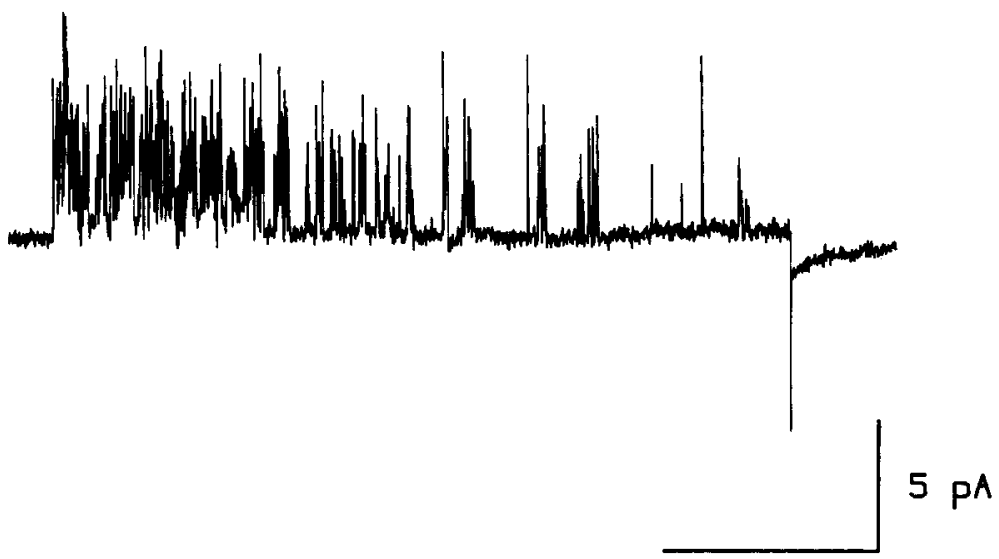

$0.5 \mathrm{~s}$
Figure 3. Voltage-dependent inactivation of single-channel currents in rod outer segments. The pipette was filled with solution $\mathrm{F}$, and the experimental chamber was perfused with solution $D$. The membrane potential is given as the negative of the voltage applied to the pipette. $A$, Outward-going unitary currents from an excised patch. The membrane potential was held at $0 \mathrm{mV}$ throughout. The data traces were obtained at an interval of $0.5 \mathrm{sec} . B$, Cellattached patch. The membrane was depolarized to $50 \mathrm{mV}$ from a holding potential of $-70 \mathrm{mV}$. Many outward unitary currents are not resolved at this low sample rate of $1000 \mathrm{~Hz}$. The data were leak-subtracted. currents increased as the membrane potential was made more positive. Most of the patches of membrane recorded from contained only a single channel, as judged by the fact that no overlapping unitary events were detected in the current records.

Figure $2 B$ illustrates currents recorded from a cell-attached patch of a rod superfused with a sodium-rich solution (solution C). The pipette holding potential was $0 \mathrm{mV}$. The actual membrane potential of the patch is unknown, but was probably -25 to $-45 \mathrm{mV}$, as discussed above. Under these conditions, depolarization of the membrane patch by $+20 \mathrm{mV}$ or more generated outward-going unitary currents. The general behavior of the outward unitary currents observed while superfusing with a sodium-rich solution was similar to that observed while superfusing with a potassium-rich solution. Thus, the currents can be observed under a conventional ionic gradient. We further characterized the outer segment currents under conditions in which the absolute membrane potential could be determined, either in cell-attached patches while superfusing with a potassium-rich solution or in excised membrane patches.

We found that if the membrane of a cell-attached or excised patch was held at a depolarized potential for a long period of time, there was a marked reduction in channel activity. This voltage-dependent inactivation had a time constant of 2-5 sec. The traces displayed in Figure $3 A$ were obtained at $0.5 \mathrm{sec}$ intervals while the membrane potential was held at $0 \mathrm{mV}$. Initially the channel showed high activity (first and second traces), but after about $1 \mathrm{sec}$ the channel activity was considerably reduced (third and fourth traces). The same behavior is shown in Figure $3 B$ on a slow time scale. In this experiment, the membrane potential was stepped to $50 \mathrm{mV}$ from a holding potential of $-70 \mathrm{mV}$. We found that the voltage-dependent reduction of channel activity occurred in both cell-attached and excised 


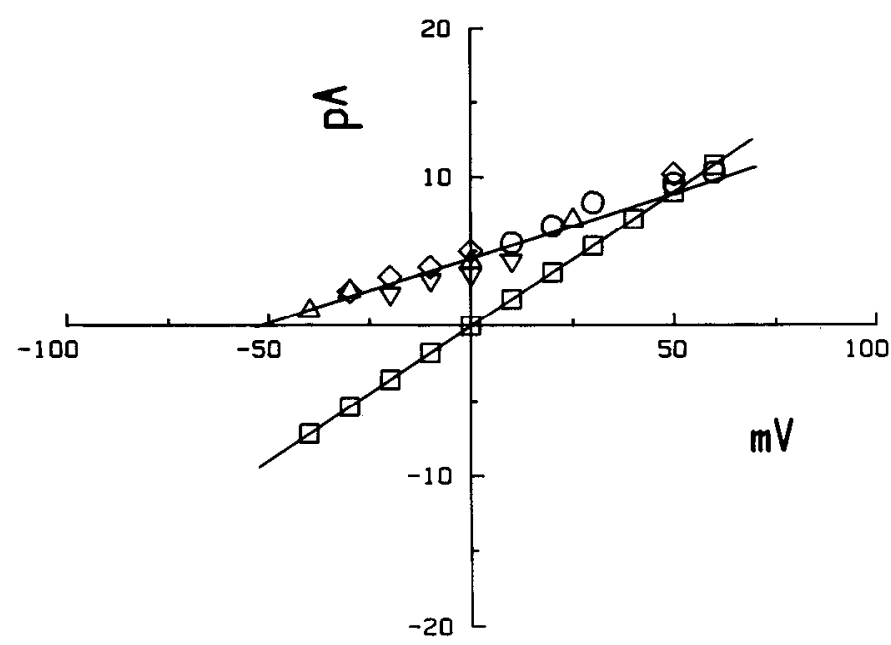

Figure 4. Single-channel current-voltage relationship. Illustrated is the amplitude of the unitary current as a function of the membrane potential. The membranc potential is given as the negative of the voltage applied to the pipette. Each data point represents an average of 20-40 amplitude determinations. The data were obtained from 5 different patches. Cell-attached patches $(\nabla, \Delta, 0, \diamond)$ were studied in rods bathed in solution $D$. Of these patches, $3(\Delta, \nabla, 0)$ were studied with the pipette filled with solution $F$, and one $(\diamond)$ was studied with the pipette filled with the same solution, but with $50 \%$ of the chloride replaced with aspartate. The reduced chloride concentration did not affect the singlechannel current amplitude or its extrapolated reversal potential. Also shown is the current-voltage relationship measured from an excised inside-out patch with equal potassium concentration on both sides of the membrane ( $\square$ ). The pipette was filled with solution $G$, and the bath solution was solution E. The circles represent measurements from a patch of inner segment membrane. A straight line was fitted to the data points of cach experiment by the lcast-squarcs method. For the experiments in the presence of low extracellular potassium concentrations, the average conductance was $87.0 \mathrm{pS}$, and the extrapolated reversal potential was $-52.5 \mathrm{mV}$. Under these conditions we did not observe inward currents. In experiments in the presence of high concentrations of potassium on both sides of the membrane $(\square)$, the conductance was $180 \mathrm{pS}$ and the reversal potential was $0 \mathrm{mV}$.

membrane patches. We have not examined the properties of inactivation further; all the records that are analyzed here were obtained with short-duration (less than $100 \mathrm{msec}$ ) voltage pulses.

Single-channel conductance. The unitary current steps that we recorded from the outer segments had short durations in both their open and closed states and were, therefore, difficult to analyze because of the limit in the high-frequency resolution of our recording system $(5 \mathrm{kHz})$. We determined the mean amplitude of the single-channel currents by averaging the amplitudes of 20-50 openings that were long-lasting. Figure 4 illustrates the current-voltage relation of single channels obtained from both cell-attached and excised, inside-out membrane patches. Recordings from excised patches were stable, provided the calcium concentration on the cytoplasmic side of the patch was not higher than $0.1 \mathrm{~mm}$. As pointed out above, results with both recording configurations were similar. When the pipette contained a low concentration of potassium (solution F), we observed outward single-channel currents, but not inward currents, because the probability of opening these channels is low at negative membrane potential (see below). At membrane potentials of -40 to $+70 \mathrm{mV}$, the conductance mechanism appeared to be ohmic, as indicated by the linear current-voltage relationship (Fig. 4). It is possible that at more negative potentials the current-voltage relation exhibits rectification, but we could not examine this possibility since we did not detect channel openings at voltages more negative than $-50 \mathrm{mV}$. The average conductance we measured for the single channel was $87 \pm$ $12 \mathrm{pS}$ (mean $\pm \mathrm{SD} ; n=4$ ).

Ionic selectivity. The straight line that was fitted to the currentvoltage relation presented in Figure 4 intersected the voltage axis at $-52 \mathrm{mV}$. This extrapolated reversal potential, given the ionic composition of the bath and the pipette, suggests that either potassium or chloride ions are the major current carrier through these channels. To distinguish between these 2 possibilities, we obtained current-voltage curves under different ionic conditions. We recorded from a cell-attached patch with a pipette filled with a solution containing half as much chloride as did the normal solution (replacing $55 \mathrm{~mm}$ chloride ion in solution $F$ with aspartate). If chloride was the charge carrier, the reversal potential should have shifted by about $17 \mathrm{mV}$. We did not, however, observe any significant change of reversal potential under these conditions (Fig. 4). In addition, if these channels were chloride-selective, it would be expected that the amplitude of the conductance would decrease as the chloride concentration in the pipette decreased. We found that the magnitude of the outward currents did not significantly change even when the concentration of chloride was reduced to $10 \%$ of its normal level.

If the outer segment channels were potassium-selective, it would be expected that the reversal potential would be $0 \mathrm{mV}$ when the membrane was exposed on both sides to solution of equal potassium concentration. Figure 5 illustrates the currents measured in an excised patch in response to a series of different voltage steps. The pipette was filled with a potassium-rich solution (solution $\mathrm{G}$ ). The patch was excised in the inside-out configuration so as to expose the cytoplasmic side to the bath solution, which consisted of isotonic potassium fluoride (KF) solution (solution E). Under these conditions, the pipette solution and the bath solution had equal concentration of potassium ions. The reversal potential was shifted to values close to $0 \mathrm{mV}$. In addition to shifting the reversal potential, elevated potassium also increased the single-channel conductance to a value of about $180 \mathrm{pS}$ (Fig. 4). These results suggest that these channels are indeed potassium-selective.

Gating. The large conductance of the potassium channels is similar to that of many types of calcium-activated potassium channels that are found in a variety of cells (reviewed by Latorre and Miller, 1983). However, the channels we describe here are not activated by $\mathrm{Ca}^{2+}$. When the cytoplasmic side of an excised inside-out patch was exposed to a solution containing low activity of calcium ions, little effect on the gating of these channels was noticed. The records displayed in Figure 5 were taken from an excised patch with the fluoride ion as the major anion on the cytoplasmic side. Under these conditions, the concentration of free $\mathrm{Ca}^{2+}$ ions was less than $10 \mathrm{nM}$, yet the activity appeared to be unaffected. The channels seem to be exclusively activated by the membrane potential.

It is apparent from the experimental records (Fig. 5) that channel activity is increased by increased membrane depolarization. We characterized the voltage-gating of these channels by calculating the open probability at different membrane potentials. The open probability was determined by dividing the mean current during a voltage pulse by the single-channel current. This procedure was carried out only with patches of membrane that contained only one channel. When patches of membrane contained only one channel, opening events did not overlap, even when the channel activity was very high. At each 

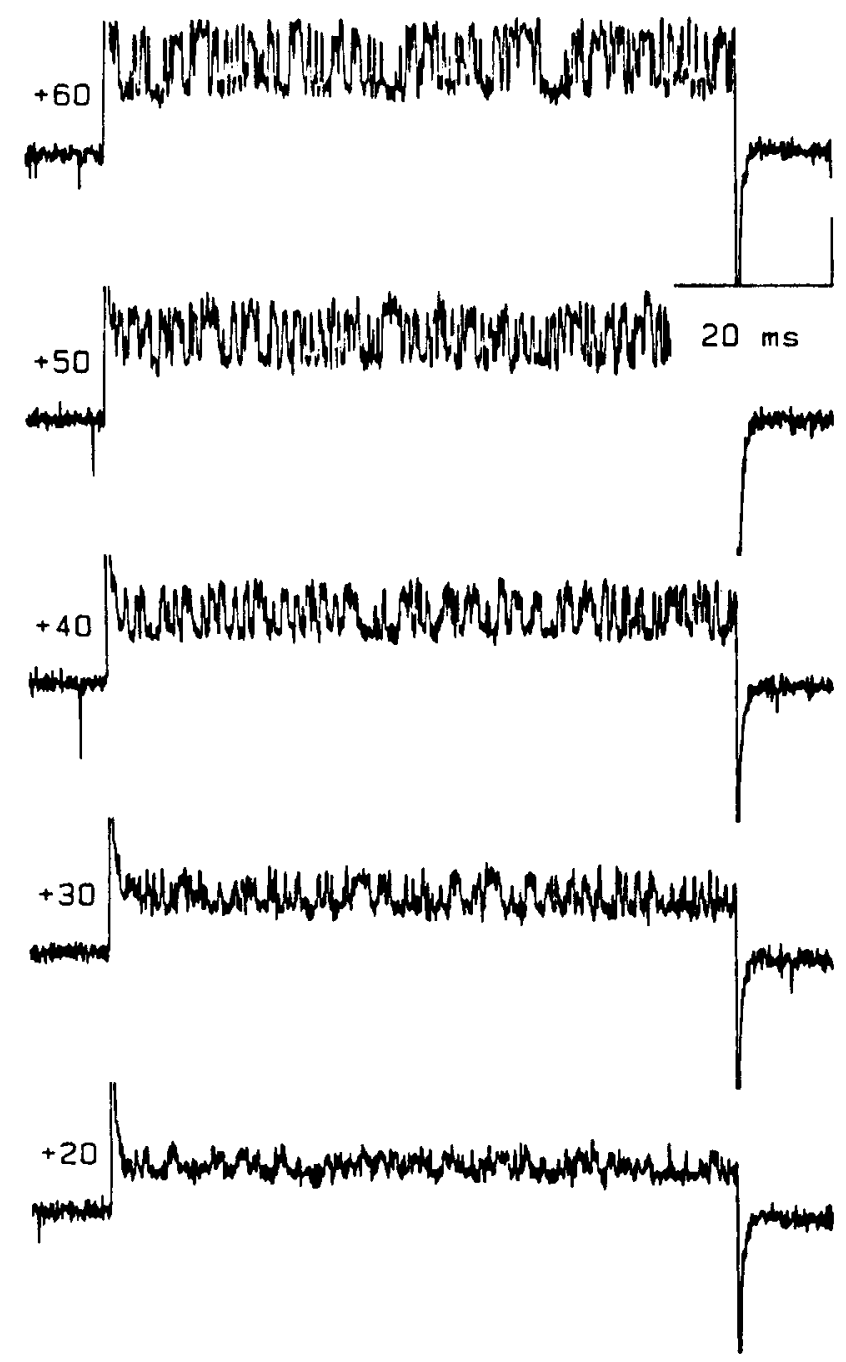

$5 \mathrm{pA}$
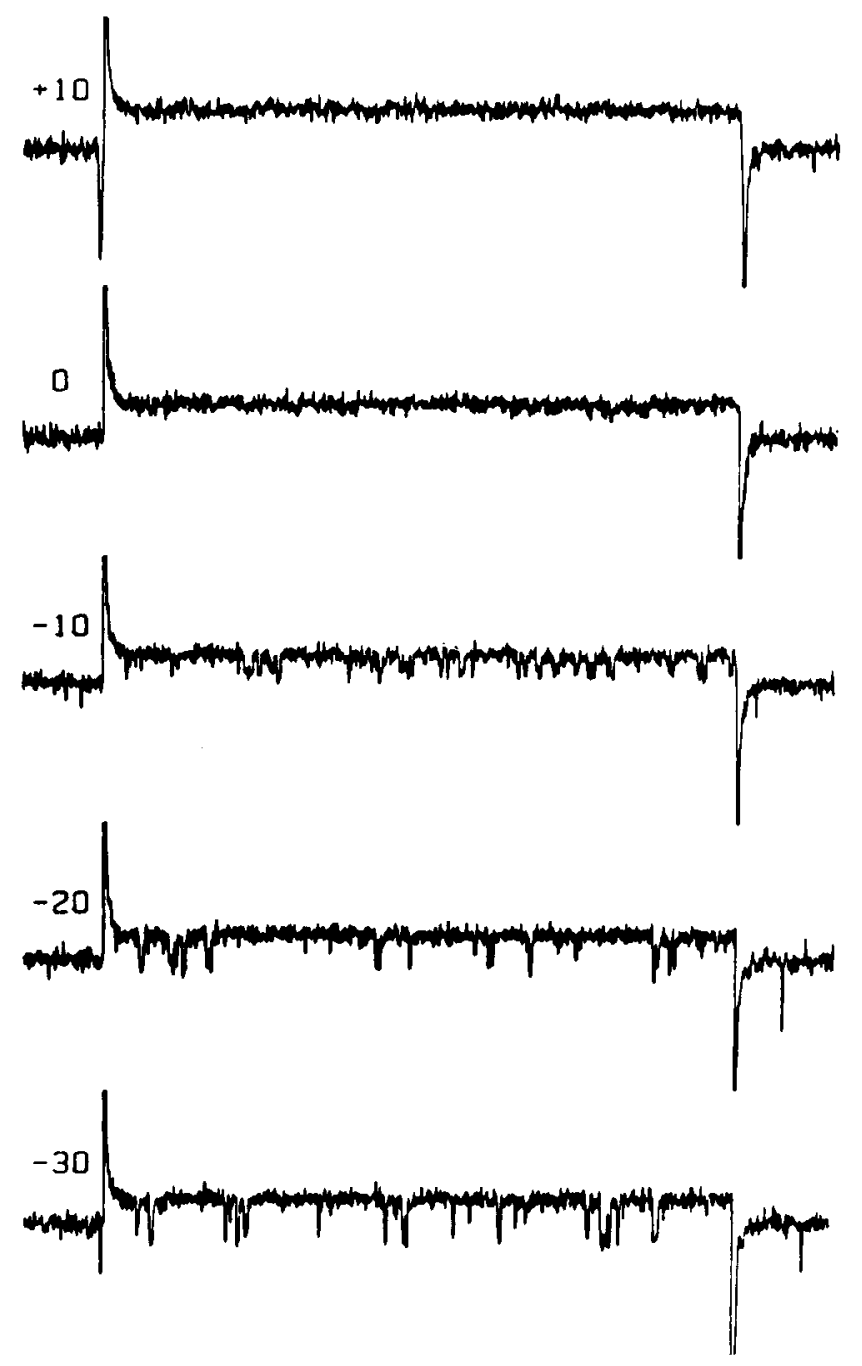

Figure 5. Unitary currents recorded from an excised inside-out patch of outer segment membrane. The pipette contained potassium-rich solution (solution $\mathrm{G}$ ) and the bath solution was isotonic potassium fluoride (solution $\mathrm{E}$ ). The membrane potential is marked on the left of each trace. The patch was held at $-50 \mathrm{mV}$ between voltage pulses. Reversal potential of the unitary currents was at about $0 \mathrm{mV}$. Note that the unitary currents are inward for $-10,-20$, and $-30 \mathrm{mV}$. The membrane potential is given as the negative of the voltage applied to the pipette, in reference to the bath voltage.

potential tested, the mean leakage current was also determined from record segments without channel activity. The single-channel current amplitude was determined from the current-voltage relation curves (Fig. 4). The fraction of the timc the channel stayed open as a function of the membrane potential for 3 patches is shown in Figure 6 . The efficiency of the membrane potential to open the channels varied widely between different patches, and in some instances we noticed changes in the voltage sensitivity in the same patch. Many patches exhibited an initial low activity that increased after a few minutes. This behavior was not due to an increase in the number of channels present in the patch, since we did not observe overlap of openings even in cases where the channel was open about $50 \%$ of the time.

The dependency of the open probability on the membrane potential that is illustrated in Figure 6 may be the result of increased closed time, decreased open time, or both. Qualitative examination of the data suggests that the effect of hyperpolarization is principally to increase the closed time. As is evident in Figure 5, the period of time between openings is increased in the less depolarized voltages. It is also possible that the open time decreased at the hyperpolarized potential, but we did not examine this possibility.

Spatial distribution. The ionic channels in rod photoreceptors are not homogeneously distributed throughout the plasma membrane (Schwartz, 1985). To study the spatial distribution of the voltage-sensitive potassium channels, described above, in the outer segments, we recorded from the inner segments of enzyme-dissociated rods. The membrane of the inner segment contains several types of voltage-dependent channels (Bader et al., 1982), and, therefore, presents a complicated pattern of electrical responses. However, in a few cell-attached patches, we observed large outward unitary currents in the inner segment membrane that resembled quantitatively the unitary currents found in the outer segment, both in amplitude and in the voltage range of activation (Fig. 4, circles). These observations suggest, then, that the channels we describe in enzyme-dissociated rods are not exclusively located in the outer segment, but are distributed in both the outer and inner segments. 


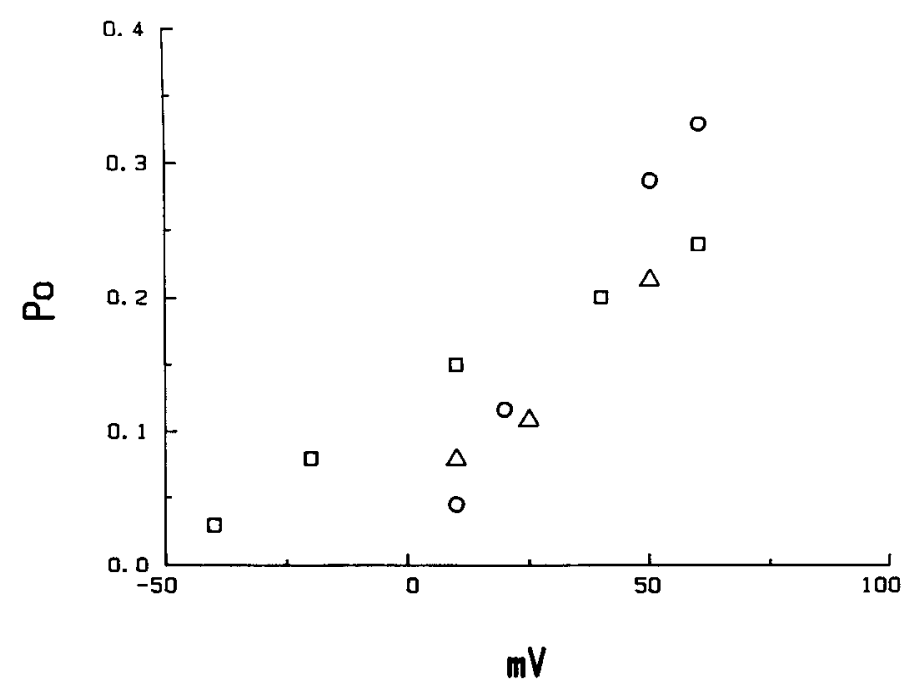

Figure 6. The channel's open probability as a function of the membrane potential. The data points were obtained by dividing the mean current by the mean amplitude of the single-channel current. The patches of membrane from which these data were collected contained only one channel, since no current overlap was observed even when the channel activity was very high. The data are from an excised patch $(\square)$ and from 2 cell-attached patches $(O, \triangle)$. Other patches showed qualitatively similar behavior. The bath solution was solution $\mathrm{D}(\triangle, O)$ or $\mathrm{E}(\square)$. The pipettes were filled with solution $F(\triangle, 0)$ or $\mathbf{G}(\square)$.

\section{Recording from mechanically isolated rods}

The contribution of the light-insensitive channels described above to the total membrane conductance depends on the number of functional channels in the membrane. We have not estimated the number of channels that are present in the outer segment membrane; however, the conductance that would be contributed by even one channel would increase the conductance of the outer segment membrane by nearly $100 \mathrm{pS}$. Yet the combined results of Baylor and Lamb (1982) and Baylor and Nunn (1986) suggest that the conductance of the outer segment membrane of light-adapted rods is less than $10 \mathrm{pS}$ over a wide range of membrane potentials, up to $\pm 50 \mathrm{mV}$. To resolve this discrepancy, we recorded from membrane patches in outer segments of rods that were isolated by mechanical means without the use of enzymes. In these experiments, we successfully obtained cell-attached recording from the tips and the sides of the outer segments of light-adapted rods. The patches of membrane were depolarized to various levels in order to activate the voltage-dependent channels. In agreement with the results of Baylor and Lamb (1982) and Baylor and Nunn (1986), we did not detect any voltage-dependent channel activity in any of the membrane patches studied ( 20 experiments). More recently, we have recorded whole-cell currents from outer segments detached from mechanically isolated rods (Hestrin and Korenbrot, 1987). In these studies, we again found no evidence of a voltage-sensitive conductance in the light-adapted preparation.

It is possible that the cells obtained by mechanical means, without the use of papain, were damaged, thus accounting for our failure to detect channels in the outer segment. We tested this possibility by mechanically isolating the rods in complete darkness and testing their response to light. Figure 7 illustrates photocurrents recorded in mechanically isolated rods. The upper panel shows records obtained from a cell-attached patch, in which the pipette (filled with solution $F$ ) made a seal on the side of the outer segment. The bottom panel illustrates whole-cell records obtained from a different cell in which the pipette (filled with solution I) was placed on the inner segment of the rod. The kinetics and light-sensitivity of the photoresponse in both configurations are similar to each other and to the photoresponses that are measured with the suction-electrode technique (Baylor and Nunn, 1986). Thus, in our hands, mechanical isolation yields normal and fully viable cells. We can conclude, therefore, that voltage-dependent and light-insensitive channels are not present in the outer segment membrane of normally functioning rods.

\section{Discussion}

We report here that the plasma membrane of the outer segments of rods obtained by enzymatic dissociation contains light-insensitive, voltage-activated potassium channels. Rods isolated by mechanical dissociation of the retina do not exhibit these channels in a form that allows their detection. Kolesnikov et al. (1984) also reported the presence of voltage-dependent channels in the rod outer segment membrane obtained from trypsindissociated cells. Noell et al. (1982) and Attwell and Gray (1984), in brief reports, also detected voltage-dependent, single-channel activity in enzyme-dissociated rod outer segments. Sather et al. (1985) found voltage-dependent channels in the outer segment membrane of rods in the absence of enzyme treatment. However, these authors noted that the occurrence of channel activity increased as the insult to normal cell integrity increased. Since we did not find any voltage-dependent channels in mechanically dissociated cells, we conclude, in agreement with Baylor and Lamb (1982) and Baylor et al. (1984), that the plasma membrane of the rod outer segment does not normally express voltagedependent, light-insensitive channels.

Our results introduce a note of caution concerning the use of enzymatic dissociation of cells. We do not suggest that enzymatic dissociation is unacceptable, or even that rods cannot be safely dissociated. Simply, cells dissociated with enzymes must be carefully examined to confirm that the spatial segregation and function of plasma membrane proteins have not been altered by the dissociation protocol. The methods of enzyme dissociation that we used here are similar to methods that have been used by Bader et al. (1982). We cannot compare our results to theirs because they only studied rods without outer segments. There are several studies using papain where the outer segment remained attached to the cell (Bader et al., 1979). However, in those studies, the methods of enzyme dissociation differed from the methods that we have used, and, perhaps more important, the currents that were measured were obtained from the entire cell membrane and cannot be specifically related to the outer or inner segment. In light of the present results it is important to compare the features of all ionic channels recorded from both enzyme-dissociated and mechanically isolated rods. The properties of the light-dependent conductance do not seem to be altered by exposure to papain (Bader et al., 1979; Baylor et al., 1984). The properties of the inward rectifier in enzyme-isolated cells (Bader et al., 1982; Bader and Bertrand, 1984) are also unaltered when compared with the inward rectifier's properties in mechanically isolated rods (Hestrin, 1987). The properties of the delayed rectifier have been studied in mechanically isolated cells by Werblin (1978) and Attwell and Wilson (1980); and in papain-isolated cells by Bader et al. (1982), the general features of that channel seem to be unaltered by the enzyme. It 

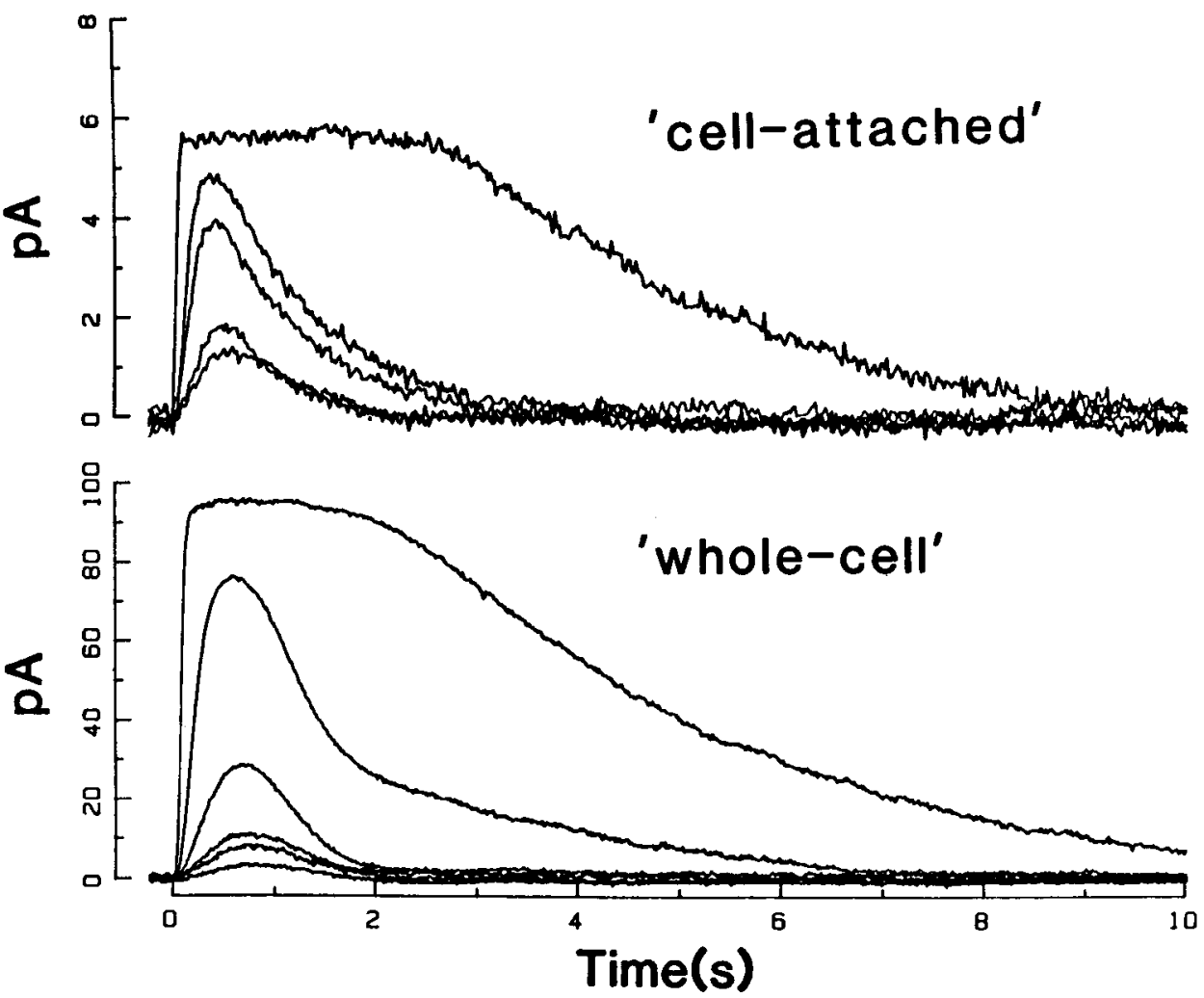

Figure 7. Photocurrent recorded from a dark-adapted rod. The cells were isolated by mechanical means. The cells were stimulated with $10 \mathrm{msec}$ flashes of $500 \mathrm{~nm}$ light. The upper panel displays photocurrents recorded in the cellattached mode from an outer segment membrane patch. The bottom panel displays whole-cell photocurrents recorded from a different cell with the pipette applied to the inner segment. The flash intensity ranged from 2 to 200 photon $\mu \mathrm{m}^{-2}$ in increments of about 2 . Cells were bathed in solution $C$. The pipette filling solution for the whole-cell recording was solution I. For the cellattached recording, the pipette was filled with solution $F$. appears, then, that, in general, the results obtained with enzymedissociated rods are not distorted by the methods of dissociation, and that the channels we report here represent a special case.

A major question regarding the present findings concerns the origin of the voltage-sensitive channels that we have detected. One possibility is that, in enzymatically dissociated cells, channels are allowed to migrate from the inner to the outer segment. In support of this possibility, Townes-Anderson et al. (1985) have recently reported that rods that are dissociated with papain are changed morphologically: the plasma membranes of the rod inner segment and outer segment are fused. Although the inner segment plasma membrane contains several types of channels, we did not find any channels in the outer segments other than the potassium-selective one reported here. We therefore do not favor the possibility of channel migration between inner and outer segments. A second possibility is that the enzyme modified the properties of the light-sensitive channel. We cannot rule out this possibility; however, we find it unlikely, because the potassium channels described here differ from the light-sensitive channels in selectivity, conductance, current-voltage characteristics, and kinetics. It would be surprising if each and every one of the channel properties would be dramatically changed by enzymatic digestion. A third possibility is that enzyme treatment interferes with the processes that segregate channels between the plasma membrane and the disk membranes, since these membranes are continuous at the base of the outer segment. Hanke and Kaupp (1984) detected, in reconstitution experiments, 2 types of voltage-activated channels derived from rod outer segment membranes. They interpreted their results to indicate that at least one of these channels is cation-selective, has a unitary conductance of $120 \mathrm{pS}$, and originates specifically from disk membrane. These channels resemble the channels that we describe here in their kinetics, mode of activation, and selectivity. It remains to be seen if the channels that are described here derive from the disk membrane. If this does prove to be the case, it would be interesting to elucidate their functional role.

\section{References}

Attwell, D., and P. Gray (1984) Patch-clamp recording from isolated rods of the salamander retina. J. Physiol. (Lond.) 351: 9p.

Attwell, D., and M. Wilson (1980) Behaviour of the rod network in the tiger salamander retina mediated by membrane properties of individual rods. J. Physiol. (Lond.) 309: 287-315.

Bader, C. R., and D. Bertrand (1984) Effects of changes in intra- and extracellular sodium on inward (anomalous) rectification in salamander photoreceptors. J. Physiol. (Lond.) 347: 611-631.

Bader, C. R., P. R. MacLeish, and E. A. Schwartz (1979) A voltageclamp study of the light response in solitary rods of the tiger salamander. J. Physiol. (Lond.) 296: 1-26.

Bader, C. R., D. Bertrand, and E. A. Schwartz (1982) Voltage-activated and calcium-activated currents studies in solitary rod inner segments from the salamander retina. J. Physiol. (Lond.) 331: 253-284.

Baylor, D. A., and T. D. Lamb (1982) Local effects of bleaching in retinal rods of the toad. J. Physiol. (Lond.) 328: 49-71.

Baylor, D. A., and B. J. Nunn (1986) Electrical properties of the lightsensitive conductance of rods of the salamander Ambystoma tigrinum. J. Physiol. (Lond.) 371: 115-145.

Baylor, D. A., G. Matthews, and B. J. Nunn (1984) Location and function of voltage-sensitive conductances in retinal rods of the salamander Ambystoma tigrinum. J. Physiol. (Lond.) 345: 203-223.

Corey, A. P., J. M. Dubinsky, and E. A. Schwartz (1984) The calcium current in inner segments of rods from the salamander (Ambystoma tigrinum) retina. J. Physiol. (Lond.) 354: 557-575.

Fain, G. L., and J. E. Lisman (1981) Membrane conductance of photoreceptors. Prog. Biophys. Mol. Biol. 37: 91-147.

Hamill, O. P., A. Marty, E. Neher, B. Sakmann, and F. J. Sigworth (1981) Improved patch clamp technique for high resolution current recording from cells and cell-free membrane patches. Pfluegers Arch. 391: 85-100.

Hanke, W., and U. B. Kaupp (1984) Incorporation of ion channels 
from bovine rod outer segments into planar bilayer. Biophys. J. 46: 587-595.

Hestrin, S. (1987) The properties and function of inward rectification in rod photoreceptors of the tiger salamander. J. Physiol. (Lond.) (in press).

Hestrin, S., and J. I. Korenbrot (1987) The effects of CGMP on the kinetics of the photocurrent in detached rod outer segments. J. Gen. Physiol. (in press).

Kolesnikov, S. S., A. L. Lyubarsky, and E. E. Fesenko (1984) Single anion channels of frog rod plasma membrane. Vision Res. 24: 12951300.

Korenbrot, J. I. (1985) Signal mechanisms of phototransduction in retinal rod. CRC Crit. Rev. Biochem. 17: 223-256.

Latorre, R., and C. Miller (1983) Conductance and selectivity in potassium channels. J. Membr. Biol. 71: 11-30.
Noell, G., E. Neher, and C. Baumann (1982) Patch electrode recordings from the plasma membrane of rod outer segments of the frog. Pfluegers Arch. (Suppl.) 394: R45.

Sather, W. A., R. D. Bodia, and P. B. Detwiler (1985) Does the plasma membrane of the rod outer segment contain more than one type of ion channel? Neurosci. Res. Suppl. 2: s89-s99.

Schwartz, E. (1985) Phototransduction in retinal rods. Annu. Rev. Neurosci. 8: 339-367.

Townes-Anderson, E., P. R. MacLeish, and E. Raviola (1985) Rod cells dissociated from mature salamander retina: Ultrastructure and uptake of horseradish peroxidase. J. Cell Biol. 100: 175-188.

Werblin, F. S. (1978) Transmission along and between rods in the tiger salamander retina. J. Physiol. (Lond.) 280: 449-470. 